\title{
Der Nachlass des Geologen Roland Strecker (1927-2007)
}

\author{
Stefan Kunze, Rolf Lange
}

Der historisch interessierte Leser kennt die Mühen der Geschichtsforschung. Versunken im Quellenmaterial der Archive sitzt der Forscher, die Zeit vergessend, still und einsam mit sich und seinem Anliegen am Tisch. Den historischen Tatsachen näher zu kommen, ist oft eine langwierige Arbeit. Gelegentlich erschließt sich durch einen Glücksfall plötzlich interessantes Material, dessen Existenz man kaum für möglich gehalten hätte. Genau ein solcher Glücksumstand ereignete sich im Jahr 2007, als Joachim Strecker aus Hohndorf, angeregt durch die Lektüre „Die Wismut in Marienberg“ von Rolf Lange, den Nachlass seines verstorbenen Bruders Roland Strecker sichtete und anschließend dem Verfasser Rolf Lange Einblick in den bergmännischen Nachlass seines Bruders gewährte. Der Nachlass erwies sich als aufschlussreich, da Roland Strecker zahlreiche Originaldokumente des Uranbergbaus (Risse, Berichte, Statistiken, Zeichnungen, Feldbücher u. a.) aus den Revieren Marienberg, Annaberg, Zobes und Schmirchau vor der Vernichtung gerettet hat. Im folgenden sollen einige Ausschnitte aus diesem Nachlass vorgestellt werden.

Tätigkeitsbezeichnung in der Wismut für einen Facharbeiter, der durch Kartierungsarbeiten geologische Daten gewinnt und diese zur weiteren geologischen Bewertung aufbereitet.
Roland Strecker 1986 im Bergbaubetrieb Schmirchau/Ronneburg

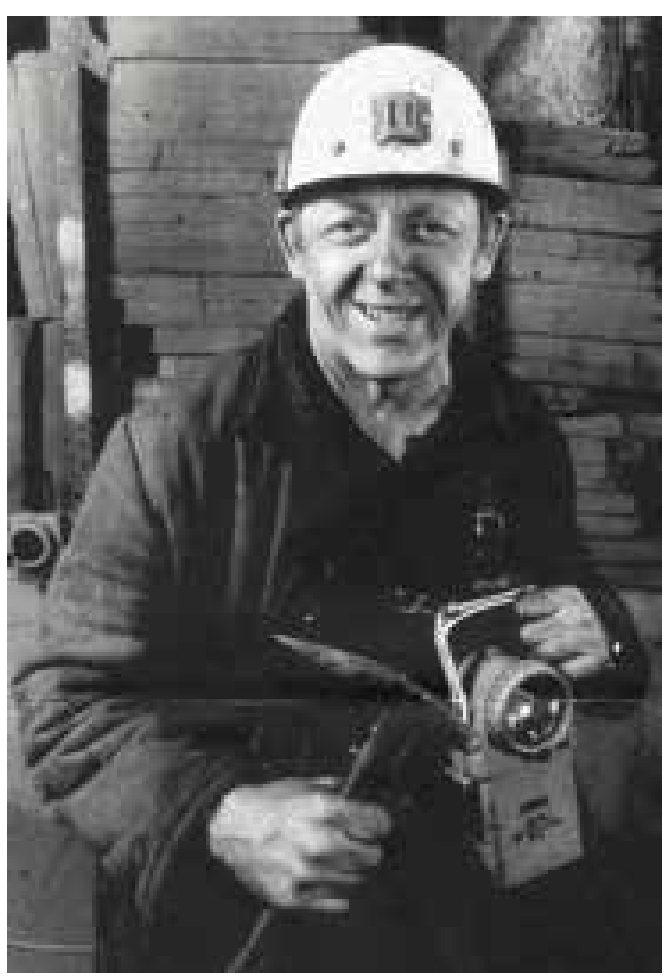

Zuvor ein paar kurze Angaben zur Person Roland Streckers. Geboren wurde er am 5. November 1927 in Schmiedeberg und starb mit knapp 80 Jahren am 24. Juni 2007 in Zwickau. Dank der gewissenhaften Nachlasspflege seines Bruders sind uns die Hauptlinien seines an Entbehrungen und Arbeitspflichten reichen Lebens bekannt. Noch als Heranwachsender zu Wehrdienst und Schanzarbeiten in Dänemark verpflichtet, verdingte sich er sich nach dem Krieg für einen Monatsverdienst von brutto 130,Reichsmark als Angestellter, Ziegelpresser und Kohlenabträger bei der Kohlengroßhandlung Franz in Zschopau. Diese harten Erfahrungen seiner Jugend formten bei ihm eine Lebenshaltung, die er später so formulierte: „Er (sein erster russischer Vorgesetzter bei der Wismut) wollte mich kontrollieren, hatte aber kein Glück, mich beim Faulenzen anzutreffen. Drill und Disziplin sowie Arbeit im Privatbetrieb hatten mich schon zu einem Arbeitstier erzogen." 1949 gibt er die chancenlose Arbeit beim Kohlen-Franz auf und fängt auf Schacht 84 in Gehringswalde beim Objekt 5 der SAG Wismut als Probenehmer an. Da er gut Zeichnen konnte, besuchte er am Bergtechnikum Freiberg einen Kollektorenlehrgang ${ }^{1}$. Später folgte noch ein Geologiestudium an der Bergingenieurschule Zwickau. Sein Arbeitsweg führte ihn über die Schächte von Marienberg, Annaberg und Zobes schließlich nach Schmirchau bei Ronneburg.

Im März 1990 ging er in Rente und verstarb 2007, wenige Monate vor seinem 80. Geburtstag in Zwickau. Die längste Zeit seines Arbeitslebens hat er als Grubengeologe bei der Wismut verbracht und galt als ein erfahrener Kenner der erzgebirgischen, vogtländischen und thüringischen Uranlagerstätten.

Der umfangreichste Teil seines Nachlasses betrifft die Lagerstätte Schmirchau und ist inzwischen Bestandteil des Geologischen Archivs der Wismut GmbH geworden (Kustos Herr Dipl.Geol. Axel Hiller). Außerdem gehören zum Nachlass kleinere, satirische Zeichnungen zum Bergbaualltag und ein Verzeichnis seiner bis auf zwei Reststücke verschollenen Mineraliensammlung. Von der Vielzahl der zum Nachlass gehörenden Dokumente soll auf ein Feldbuch näher eingegangen werden, dessen Inhalt die damali- 


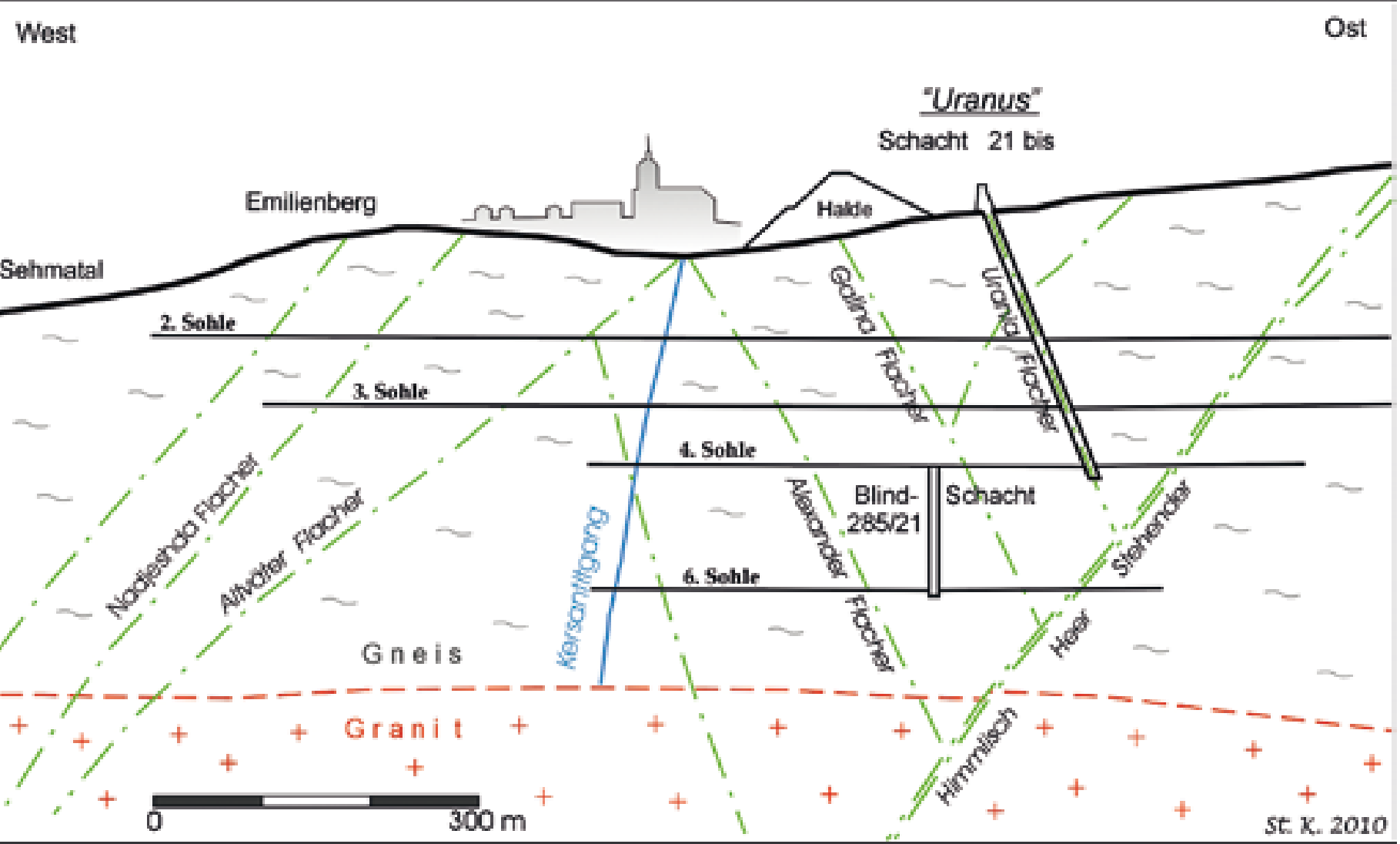

gen Arbeitsprozesse auf eine sehr persönliche Art und Weise wiedergibt. Für den Grubengeologen stellt das Feldbuch ein wichtiges Arbeitsmittel dar. Darin werden die vor Ort gewonnenen Erkenntnisse grafisch und textlich festgehalten, um sie später ins geologische Risswerk des Schachtes übertragen zu können. Nach einer Vermittlung des persönlichen Kontakts zu Joachim Strecker durch den Verein vom Besucherbergwerk Markus Röhling wurde mir (St.K.) in dankenswerter Weise das Feldbuch von Roland Strecker zur Auswertung überlassen.

Bei diesem Feldbuch handelte es sich um einen Block aus 73 Blatt Millimeterpapier im Format
A6 in einer robusten Papphülle. Die darin auf den Vorder- und Rückseiten mit Bleistift getätigten Eintragungen zeigen geologische Kartierungsarbeiten vom 16. Mai bis zum 7. Juli 1954. Zunächst bestand noch Unklarheit über die Örtlichkeit der Dokumentationen, da weder Schacht noch Lagerstätte explizit vermerkt waren. An Hand von Grubenbaubezeichnungen konnte schließlich eine zweifelsfreie Identität mit tatsächlich im Jahre 1954 auf oberen Sohlen des Annaberger Schachtes „Uranus“ belegten Betriebspunkten nachgewiesen werden. Dieses Feldbuch spiegelt nicht nur rein geologische und bergbautechnologische Aspekte des

\begin{tabular}{|l|l|l|l|l|}
\hline \multicolumn{5}{|c|}{ Mai 1954} \\
\hline Mo & 10 & 17 & 24 & 31 \\
\hline Di & 11 & 18 & 25 & \\
\hline Mi & 12 & 19 & 26 & \\
\hline Do & 13 & 20 & 27 & \\
\hline Fr & 14 & 21 & 28 & \\
\hline Sa & 15 & 22 & 29 & \\
\hline So & 16 & 23 & 30 & \\
\hline
\end{tabular}

Geologischer Schnitt vom Annaberger Schacht 21 „Uranus" Roland Strecker arbeitete hier in den Jahren 1953 bis 1954 Quelle: Arbeitsprojekt des Objektes 111 für das Jahr 1955, umgezeichnet
Kalendarische Auswertung des Feldbuchs blau - Tage mit Feldbucheinträgen 27. Mai - Christi Himmelfahrt 07. Juni - Pfingstmontag 14. bis 20. Juni - evtl. Urlaubswoche 
Angaben zur Gesteinskategorie: dichter Gneis Kategorie 9 (Anteil $50 \%$ ), Flasergneis Kategorie 8 (Anteil $50 \%$ )

Etagenstrecke 508 Nord oben 28. Juni 1954, Feldbuch Blatt 56

Ortsbrust einer Strecke (Scheibenbild): „Rechter Teil: Hauptgang $\mathrm{CaF}_{2}$ violett, Tschörn [von russ.: "чёрнь" - Uranschwärze], $\mathrm{SiO}_{2}$, $\mathrm{Fe}_{2} \mathrm{O}_{3}, \mathrm{Mg} \mathrm{Ca}$ [Dolomit] zersetzt, im Hangenden Letten, grau Linker Teil: Tschörn; $\mathrm{SiO}_{2} ; \mathrm{Fe}_{2} \mathrm{O}_{3} ; \mathrm{CaF}_{2}$ Gneis unten: flasrig angewittert, Limonit, Schwebender: in 2

Trümern à $3-5 \mathrm{~cm}$, schwarze Letten, Gneisruschel" Strecke 40 a Süd auf „Gang Nadjeshda" 16. Mai 1954, Feldbuch Blatt 2

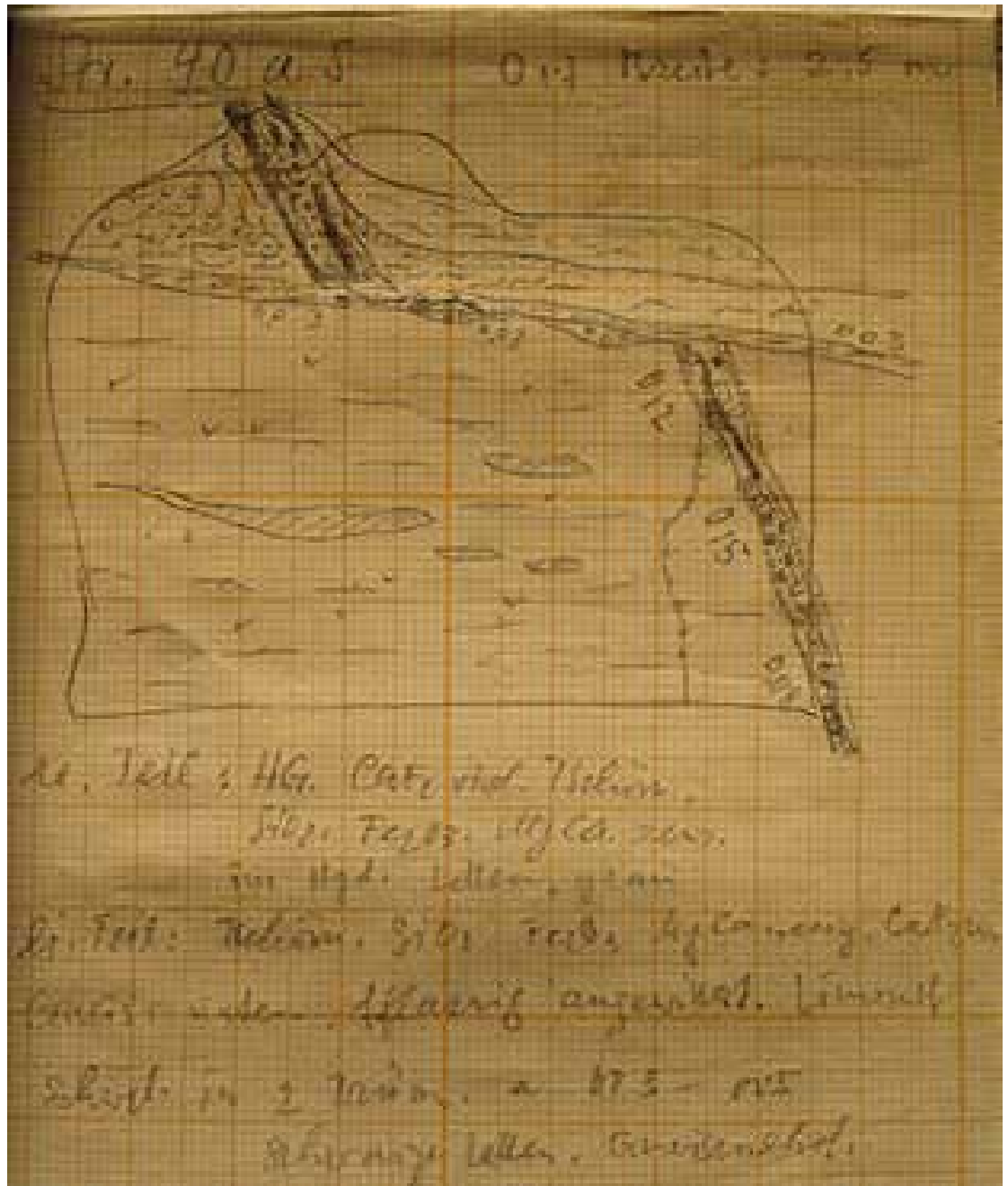

damaligen Uranbergbaus wieder. Im Geologischen Archiv der Wismut GmbH ist für die Lagerstätte Annaberg ein relativ großer Teil des geologischen Risswerks archiviert. Die hingegen kaum noch erhaltenen Feldbücher zeigen jedoch direkt den Umfang an geleisteter Feldarbeit der Grubengeologen auf, wie er umfassend aus den vorgenannten, generalisierten Quellen nicht mehr nachzuvollziehen ist. Beispielsweise ist das Verhältnis der untertägigen Feldarbeit zur Büroarbeit (diese bestand u. a. im Nachtragen des geologischen Risswerkes) an Hand der kalendarischen Auswertung der Feldbuchdaten ersichtlich. Das erste Dokumentationsdatum fällt z. B. auf einen Sonntag, an dem zu damaliger Zeit teilweise auch noch gearbeitet wurde. Ansonsten weisen die Angaben im Feldbuch auf die damals übliche 6-Tage-Woche sowie auf einen bis 1967 arbeitsfreien kirchlichen Feiertag hin (Christi Himmelfahrt).

Am Beispiel der Lagerstätte Annaberg lässt sich der Einfluss des jeweiligen lagerstättengeologischen Erkenntnisstandes auf die wechselhafte Entwicklung von Gewinnung und Umfängen des Bergbaus besonders deutlich erkennen. Ebenbürtig mit allen anderen bergbautechnologischen Berufsgruppen ist deshalb auch die explodierten förmlich die Auffahrungsumfänge, ohne dass der geologische Dienst in seinem Personalbestand an qualifizierten Mitarbeitern Schritt halten konnte. So betrugen im Jahr 1949 die Auffahrungsumfänge mindestens das Zehnfache des Wertes von 1947, allerdings steigerte sich die Erzgewinnung der Schächte nicht in diesem Maße, sondern nur etwa auf das Sechsfache. Nach einem dramatischen Tiefpunkt der Erzgewinnung um 1951 entdeckte man während des Folgejahres schließlich eine ausgeprägte Affinität der Gangvererzung zu bestimmten Gesteinsschichten. Natürlich war dieses Phänomen auch schon vorher lokal bekannt, ist aber aus verschiedenen Gründen für die Gesamtbetrachtung der Lagerstätte weitgehend unbeachtet geblieben. Aus dieser Erkenntnis ergaben sich zwangsläufig neue Maßstäbe für die geologische Betreuung der Bergarbeiten. Die Strecken und Überhauen der Vorrichtung ${ }^{2}$ sollten sich nunmehr streng innerhalb der flach einfallenden produktiven Gesteinspakete ${ }^{3}$ bewegen, um unnötige Auffahrungen zu vermeiden. Bis zum Ende des Annaberger Uranbergbaus waren deshalb die Grubengeologen angehalten, bei der Durchsetzung dieser Anweisungen im Rahmen ihrer fachlichen Kompetenz maßgeblich mitzuwirken. Dass diese Maßnahmen auch zu einem Erfolg führten, zeigten die bis zum Ende der Bergarbeiten im ersten Quartal des Jahres 1958 stetig steigenden Effektivitätskennziffern ${ }^{4}$.

Allein wie dies geschah, ist aus den Inhalten des Feldbuches nur zu gut ersichtlich. Etwa 250 Dokumentationen von Vortriebsörtern (Scheiben- 


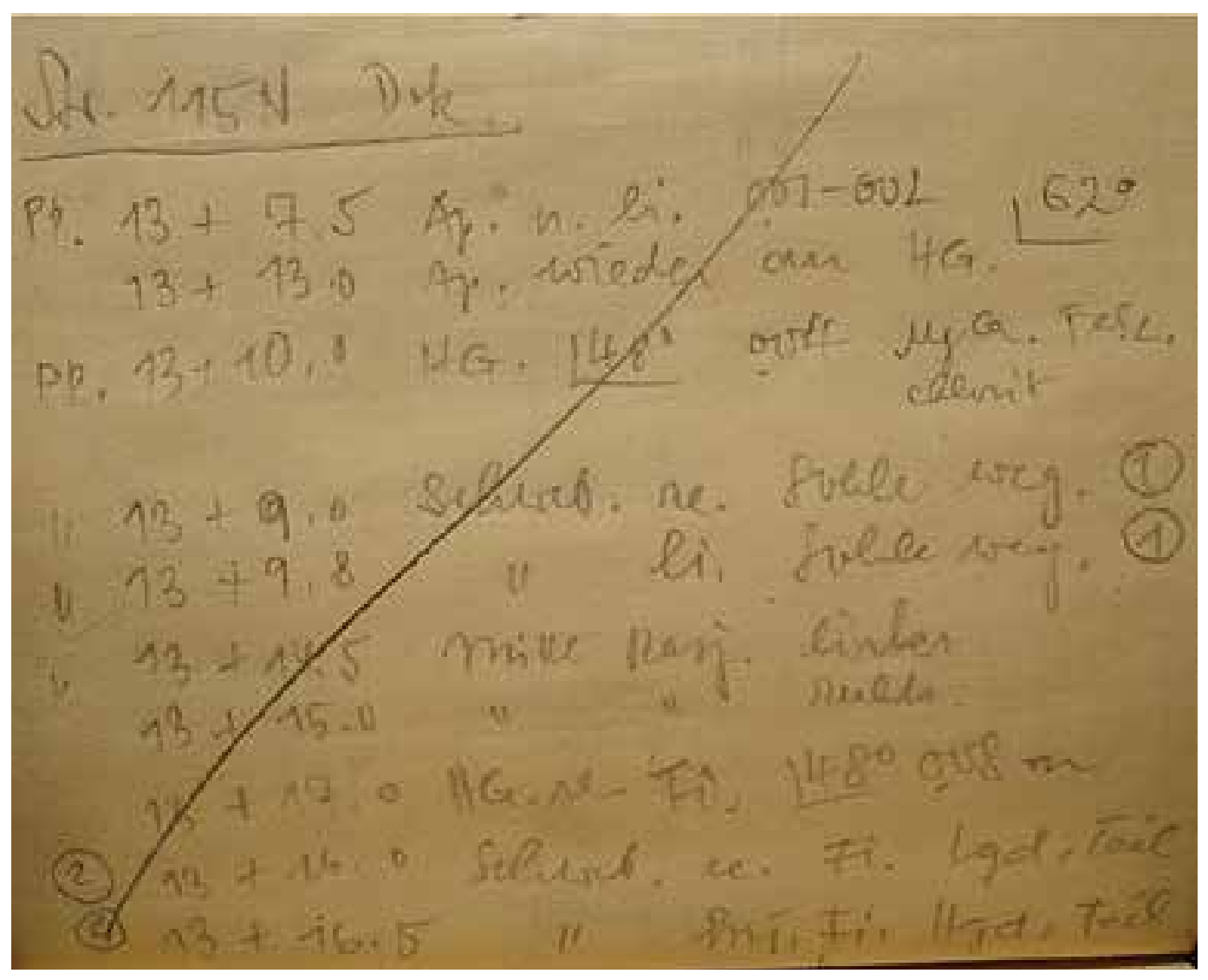

bilder, Stoßaufnahmen etc.) enthalten oft konkrete Anweisungen für die unmittelbar folgenden Arbeiten, zumeist Richtungs- und Profilkorrekturen sowie Auffahrungseinstellungen, wenn man den erzhöffigen Bereich durchörtert hatte.

\section{Gesteinsklassifizierung:}

Bohrbarkeit von Gesteinen (umgangssprachlich im Feldbuch auch als „Härtegrad“ bezeichnet), nach der Anlage zum Befehl Nr. 79 vom 24. März 1951 des Generaldirektors M. Malzew als Normierungsgrundlage für Bergbauarbeiten und Tiefenbohrungen auf einer Skala von $12 \mathrm{Ge}-$ steinskategorien.

\section{Kategorie 1:}

Sandböden mit Geröll und Schotter, etc.

\section{Kategorie 12:}

Mikrogranite, Syenite, etc.

Im Feldbuch erscheinen durchweg die Kategorien 8 und 9, überwiegend in Zehnerprozenten von $20 \%$ bis $80 \%$ abgestuft.

\section{Kategorie 8:}

z. B. quarzarmer Zweiglimmergneis Bohrzeit: 7,1 - 11,0 Minuten / Bohrmeter

\section{Kategorie 9:}

z. B. quarzreicher Zweiglimmergneis Bohrzeit: 11,1 - 19,0 Minuten / Bohrmeter
Des Weiteren war bei der Festlegung der Gesteinskategorie (Bohrhärte) eine Zuarbeit des Geologen für die Normabteilung gefragt, wie sich an diesen häufigen Eintragungen im Rahmen der Ortsaufnahmen zeigt. Als methodisch interessanten Aspekt konnte man bei den geologischen Aufnahmen zwei verschiedene Kartierungsarten feststellen. Zum einen die Ortsaufnahmen, welche aus Scheibenbildern, seltener Stoß- und Firstkartierungen der letzten Auffahrungsmeter bestehen. Hierbei ist nur in den seltensten Fällen der Vortriebsstand (Auffahrungslänge, Abstand $\mathrm{zu}$ Festpunkten) vermerkt. Dessen lagemäßige Zuordnung für eine Verwendung dieser Daten im geologischen Risswerk konnte offenbar nur sehr grob über den markscheiderischen Dekaden- und Monatsvortriebsstand erfolgen. Somit lag der Schwerpunkt dieser Ortsaufnahmen eher im Erfassen der aktuellen geologischen Situation und dem Treffen von operativen Entscheidungen für die unmittelbar bevorstehenden Bergarbeiten. Aus den Angaben des Feldbuchs ist ersichtlich, dass pro Schicht 5-23 solcher Ortsaufnahmen gefertigt wurden. Die andere Kartierungsart bestand in der Doku-

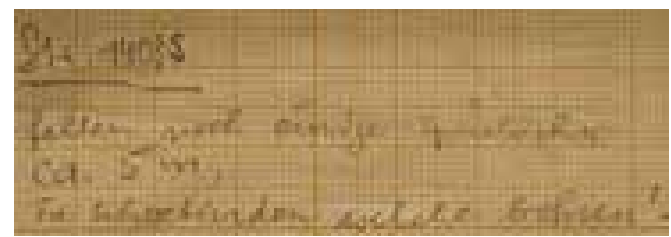

Dokumentation in Textform ausgehend von einem Polygonpunkt (PP 13)

Auszug: „PP $13+7,5 \mathrm{~m}$ Apophyse nach links 1-2 cm mächtig, Einfallen $62^{\circ}$

PP $13+13,0$ m Apophyse wieder an den Hauptgang geschart PP $13+10,0 m$ Hauptgang Einfallen $48^{\circ}$, Dolomit, Pyrit, Chlorit", Strecke 115 Nord 8. Juni 1954, Feldbuch Blatt 31

2 Bergmännische Vorbereitungsarbeiten (Горно-подготовительные работы, ГПР): Grubenbaue, welche die Lagerstätte abbauvorbereitend aufschließen.

3 Gneis mit einer Häufung von geringmächtigen Metasapropelitlagen (graphit- und sulfidführend, in strukturell-tektonischer Prägung zumeist als „Schwebende" bezeichnet)

4 Verhältnis zwischen den $\mathrm{Ge}$ winnungs- und Aufwandsdaten der Bergbautätigkeit (Metallmenge des gewonnenen Erzes zur aufgefahrenen / abgebauten Gangfläche).

5 Abgewandelte Transkription des russ. Begriffes ,шпур“ (Spur) für Sprengbohrloch.

6 Abgeleitet von russ.: „каротаж“: Bohrlochmessung mit geophysikalischen Methoden, hier Messung der Gammaexpositionsleistung als Indikator des Urangehaltes. Der russ. Begriff entstammt (unter Abwandlung der Bedeutung) ursprünglich von franz.: „carottage“ - Kernbohrung.

Anweisung zur Einbringung der Kontrollbohrung:

„fehlen noch einige Spürlöcher ca. $5 \mathrm{~m}$, in Schwebenden welche bohren!" Strecke 140 Süd 28. Mai 1954, Feldbuch Blatt 16 


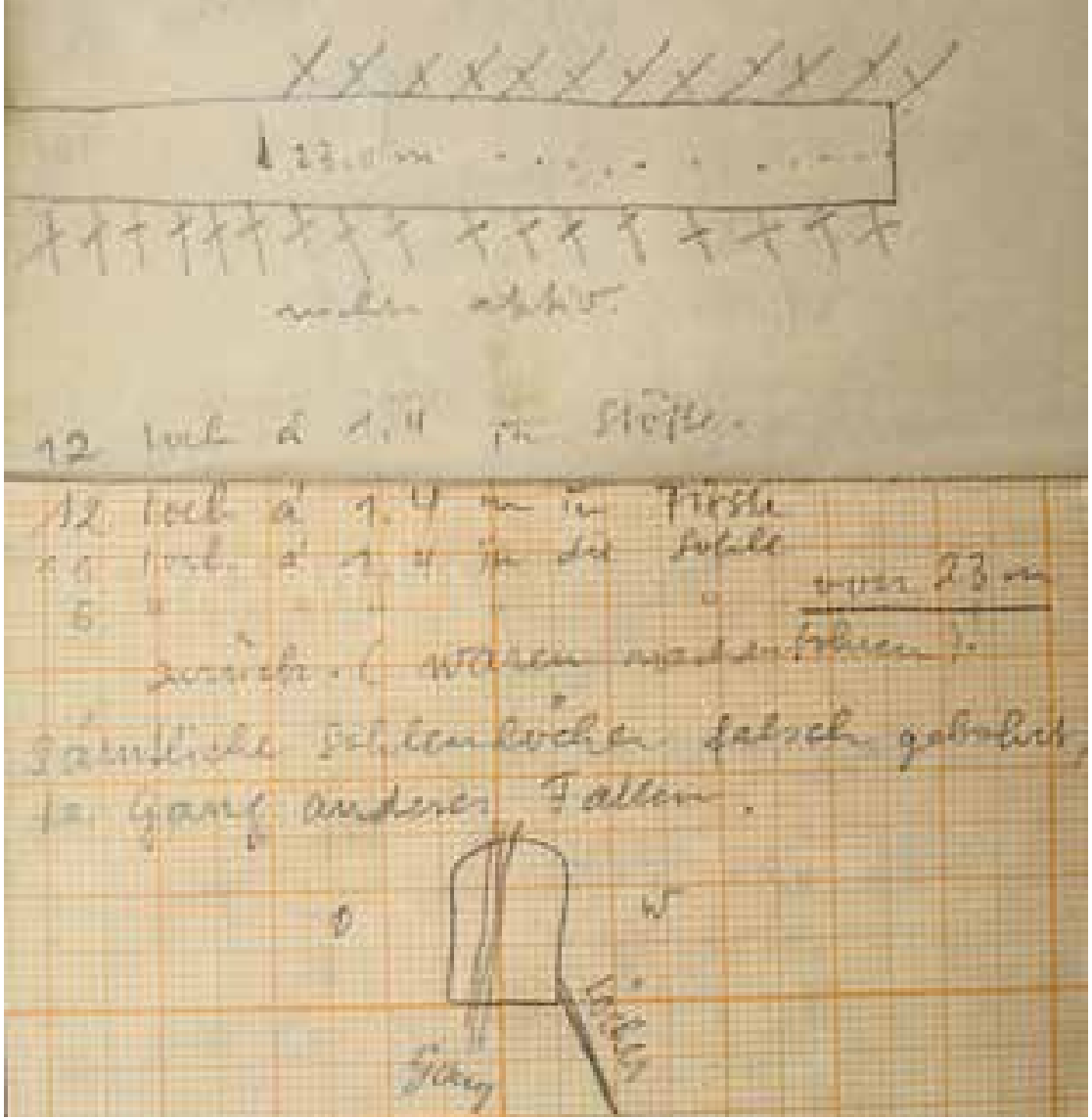

Karottageaufnahme mit geologischer Beurteilung der Bohrqualität: „6 Loch a 1,4 m in die Sohle von $23 \mathrm{~m}$ zurück (waren nachzubohren). Sämtliche Sohlenlöcher falsch gebohrt, da Gang anderes Fallen."

Etagenstrecke 800 unten

16. Mai 1954, Feldbuch Blatt 3 und 4

Skizze der Gamma-Karottage: "13 Loch a 1,3 m Stöße

13 Loch a $1,4 \mathrm{~m}$ Sohle

11 Loch a 1,3 m Firste. Summe 379 Loch mit Erz"

(Zahlen 1-4 = Erzsorten, A = aktiv) Etagenstrecke 106/10

31. Mai 1954, Feldbuch Blatt 20 und 21

Autoren

Stefan Kunze Sehmatal

Rolf Lange Drebach mentation, welche im Feldbuch immerhin 21 Blatt einnimmt und mit dem Kürzel „Dok.“ bezeichnet wurde. Hierbei erfolgte die geologische Dokumentation eines längeren Auffahrungsab-

Die Parameter der geologischen Strukturen wurden mit genauen Lageangaben (Entfernung zu Polygonpunkten, Überhauen, Pikett) textlich verzeichnet. Grafische Aufnahmen erfolgten nur bei komplizierten Verhältnissen, die ohne Skizze schwer zu beschreiben waren. Die damals schon auf verschiedenen Schächten übliche, zeitaufwändigere Dreiseitenkartierung (grafische geologische Aufnahme beider Stöße + Firste) wurde nicht praktiziert. Aus der (Primär-) Dokumentation erstellten die kartierenden Geologen eine grafische Sekundärdokumentation.

Die Daten der Sekundärdokumentation flossen nach einer Generalisierung schließlich ins geologische Risswerk ein, welches für einen langfristigen Zeitraum als Planungsgrundlage der Bergarbeiten diente. Außerdem oblag den Geologen die Anweisung und Beurteilung der durch den Bereich Geophysik radiometrisch abzunehmenden Kontrollbohrungen geringer Tiefe (1,2-1,5 m), welche in Firste, Sohle und Stöße der Gangauffahrungen einzubringen waren. Im Feldbuch werden sie als „Spürlöcher“5 bezeichnet; das unmittelbare Umfeld der Grubenbaue wird damit auf Erz „abgespürt". Die geologische Beurteilung beinhaltete eine Kontrolle von First- und Sohlenlöchern auf schnittes $(50-100 \mathrm{~m})$.

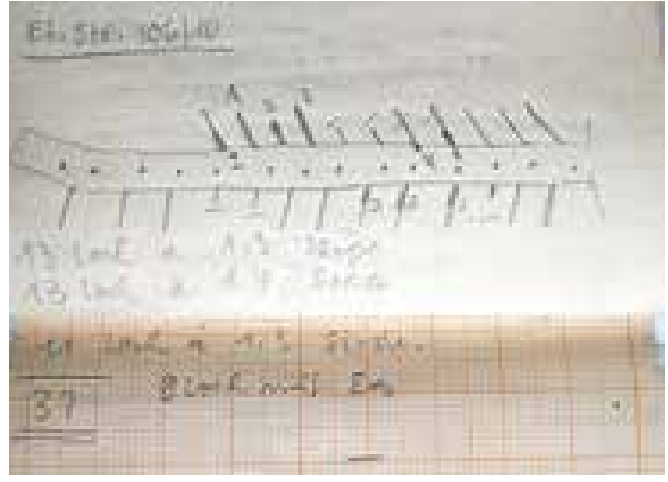

lagerichtige Ausführung zur Gangfläche. Falsch gebohrte Löcher waren nachzubohren. Es finden sich im Feldbuch auch einige wenige Gamma-Karottageskizzen ${ }^{6}$ mit Eintragung der Erzsorten und genauer Abrechnung von Bohrlochanzahl und Bohrlochtiefe (Bezahlungsgrundlage der Hauer), die eigentlich zum Aufgabenbereich der deutschen Geophysik-Operatoren gehörten.

Angaben zum gewonnenen Erz sind ebenso nur sporadisch aufgeführt (Kistenanzahl/Erzsorte). Möglicherweise hat er diese Orte zusammen mit einem Geophysik-Operator befahren und dessen Feststellungen dokumentiert.

Mit streng geheimen Produktionsdaten (gewonnene Metallmenge, Vorratsberechnungen) kam Roland Strecker nicht in Berührung, diese lagen ausnahmslos in der Hand des sowjetischen Personals. Er war somit einer der vielen deutschen Geologen, zu deren Aufgabengebiet umfangreiche Kartierungen sowie die unmittelbare geologische Betreuung der Bergarbeiten gehörten.

Für die Erforschung des Uranbergbaus in der Lagerstätte Annaberg stellt das Feldbuch von Roland Strecker ein unikates Zeitdokument dar, dessen Auswertung interessante Details der damaligen Situation beleuchten konnte. Es wäre zu wünschen, dass weitere in Privatbesitz befindliche Dokumente des regionalen Uranbergbaus in kompetente Hände gelangen, damit deren fundierte Auswertung ermöglicht werden kann.

Quellen:

Informationen von Herrn Joachim Strecker, Hohndorf b. Zschopau.

Biografische Fragmente, Nachlass Roland Strecker.

Feldbuch, Schacht 21 Annaberg, Nachlass Roland Strecker.

Wismut GmbH, Geologisches Archiv: Jahresberichte und Arbeitsprojekte der Lagerstätte Annaberg.

Stefan Kunze: Die Schwebenden im Südteil der Ganglagerstätte Annaberg Teil 1 und 2. (Weisbachiana-Hefte Nr. 19 und 20), Arnsfeld 2005.

Stefan Kunze, Frank Langer: Die Entwicklung des Uranbergbaus der SAG/SDAG Wismut in der Lagerstätte Annaberg. -Tagungsband 14. Internationaler Montanhistorik Workshop, Annaberg-Buchholz 2011.

Internet www.wismut.su: Erinnerungsbericht des Geologen I.I. Tolmatschow (letzter Zugriff November 2016). 